\title{
Isolation of the highly pathogenic and zoonotic agent Burkholderia pseudomallei from a pet green Iguana in Prague, Czech Republic
}

\author{
Mandy C Elschner ${ }^{1 *}$, Jan Hnizdo ${ }^{2}$, Ivonne Stamm³ ${ }^{3}$ Hosny El-Adawy ${ }^{1}$, Katja Mertens ${ }^{1}$ and Falk Melzer ${ }^{1}$
}

\begin{abstract}
Background: Melioidosis caused by Burkholderia (B.) pseudomallei is an endemic zoonotic disease mainly reported from northern Australia and Southeast Asia. In Europe, cases of human melioidosis have been reported only from patients travelling to endemic regions. Besides humans, B. pseudomallei has a very broad host range in domestic and wild animals. There are some reports about importation of B. pseudomallei-infected animals from endemic areas into Europe. The present report describes the first case of B. pseudomallei infection of a pet iguana in Europe.

Case presentation: In a 5-year-old pet Iguana iguana living in a private household in Prague, Czech Republic, B. pseudomallei was isolated from pus of an abscess. The isolate VB976100 was identified by Vitek ${ }^{\circledR}$, MALDI-TOF mass spectrometry and polymerase chain reaction as B. pseudomallei. The molecular typing resulted in multi-locus sequence type 436 hitherto, which has been found only once worldwide in a B. pseudomallei strain isolated in the USA and originating from Guatemala. The identification as internal transcribed spacer type G indicates a close relatedness to strains mainly isolated in the Western Hemisphere. These findings support the hypothesis that the iguana became infected in this region or in a breeding facility through contact to other infected animals.
\end{abstract}

Conclusions: The present case highlights the risk of importation of the highly pathogenic and zoonotic B. pseudomallei into non-endemic regions through animal trade. Therefore, veterinarians treating animals from these areas and physicians examining patients owning such animals should include melioidosis in differential diagnosis whenever specific symptoms appear. Furthermore, veterinary authorities responsible for supervision of traders and pet shops should be aware of this risk of zoonotic transmission.

Keywords: Melioidosis, Burkholderia pseudomallei, Zoonoses, Iguana

\section{Background}

Burkholderia (B.) pseudomallei is the causative agent of melioidosis, an endemic disease mainly reported from northern Australia and Southeast Asia. In this area the disease has emerged as an important cause of morbidity and mortality during the last 25 years [1]. Endemic and sporadic cases of melioidosis are also reported from countries of South America, North America, Oceania, as well as Aruba, Guadeloupe, Guam, Haiti, Martinique, and Puerto Rico. Increasing numbers of cases are reported from Africa [2]. However, in Europe cases of human

\footnotetext{
* Correspondence: mandy.elschner@fli.bund.de

'Friedrich-Loeffler-Institut, Federal Research Institute for Animal Health, Institute of Bacterial Infections and Zoonoses, Naumburger Strasse 96a, 07743 Jena, Germany

Full list of author information is available at the end of the article
}

melioidosis have only reported from patients who had been travelling to endemic regions [3,4]. The gramnegative bacterium belongs to biohazard risk group 3 agents, is listed as biowarfare agent, and has to be processed under biosafety level 3 conditions. The disease is mainly acquired environmentally by percutaneous infection, ingestion, or inhalation. The incubation period of melioidosis can vary between one day and 62 years, depending on the route of infection and infection dose [1]. Clinical studies have shown that pneumonia is the predominant clinical manifestation, followed by skin and soft tissue infections, as well as acute suppurative parotitis, especially in pediatric cases, and prostatitis in males [5]. One predominant risk factor is diabetes mellitus, as shown by studies in Australia and Thailand, where up to $60 \%$ of the melioidosis patients are diabetic, mainly type 2 [5]. 
Besides humans, B. pseudomallei has a very broad host range. In domestic animals it is most commonly reported in cattle, goats and swine [6]. However, sporadic cases or small outbreaks have been reported in monkeys, gibbons, orangutans, kangaroos, wallabies, deer, buffaloes, cows, camels, llamas, zebras, koalas, dogs, cats, horses, mules, parrots, rats, hamsters, rabbits, guinea pigs, ground squirrels, seals, dolphins, crocodiles [7]. Very recently, $B$. pseudomallei infections in two pet iguanas in California were reported [8]. In animals, acute and chronical form of melioidosis is seen. Common symptoms in animals include anorexia, pyrexia, coughing, skin dehydration and abscesses $[7,9]$.

\section{Case presentation}

The female Iguana iguana was purchased by private owners as a young animal from a pet shop in Prague in 2009. It was kept alone in a terrarium, but was also allowed to move freely outside the cage in the flat (Figure 1). The owners had a very close and tender contact to the animal, such as hand feeding and kissing. Usually the iguana was fed with special pellet formulation for iguanas, fresh green salad, vegetables and fruits, and once a month by
Zophobas morio larvae purchased from different breeders. In 2011, the animal developed an abscess underneath the left eye, which was transected and treated antibiotically without microbiological investigation. In 2013, the animal was admitted to the Animal Clinic, Bila Hora, Prague, Czech Republic, where again an abscess was diagnosed, this time at the root of the tongue. After surgical intervention and antibiotic treatment by marbofloxacine the animal recovered. One year later, in 2014 the owners presented the animal again to the clinic because of a weeklong anorexia. The clinical investigation resulted again in an encapsulated abscess located at the root of the tongue. This time the abscess was sampled for microbiological analysis. As soon as the first suspicion of a B. pseudomallei infection was announced, the veterinary authority was informed, and it mandated the immediate euthanization and incineration of the animal. One veterinary assistant was bitten by the iguana when he tried to fix the maxilla for sampling of the abscess by the veterinarian. The skin was injured although he wore gloves. Finally, the wound was cured and the assistant was prophylactically treated with amoxicillin-clavulanate for 10 days in a clinic specialized for infectious diseases. Also the owners, not showing any

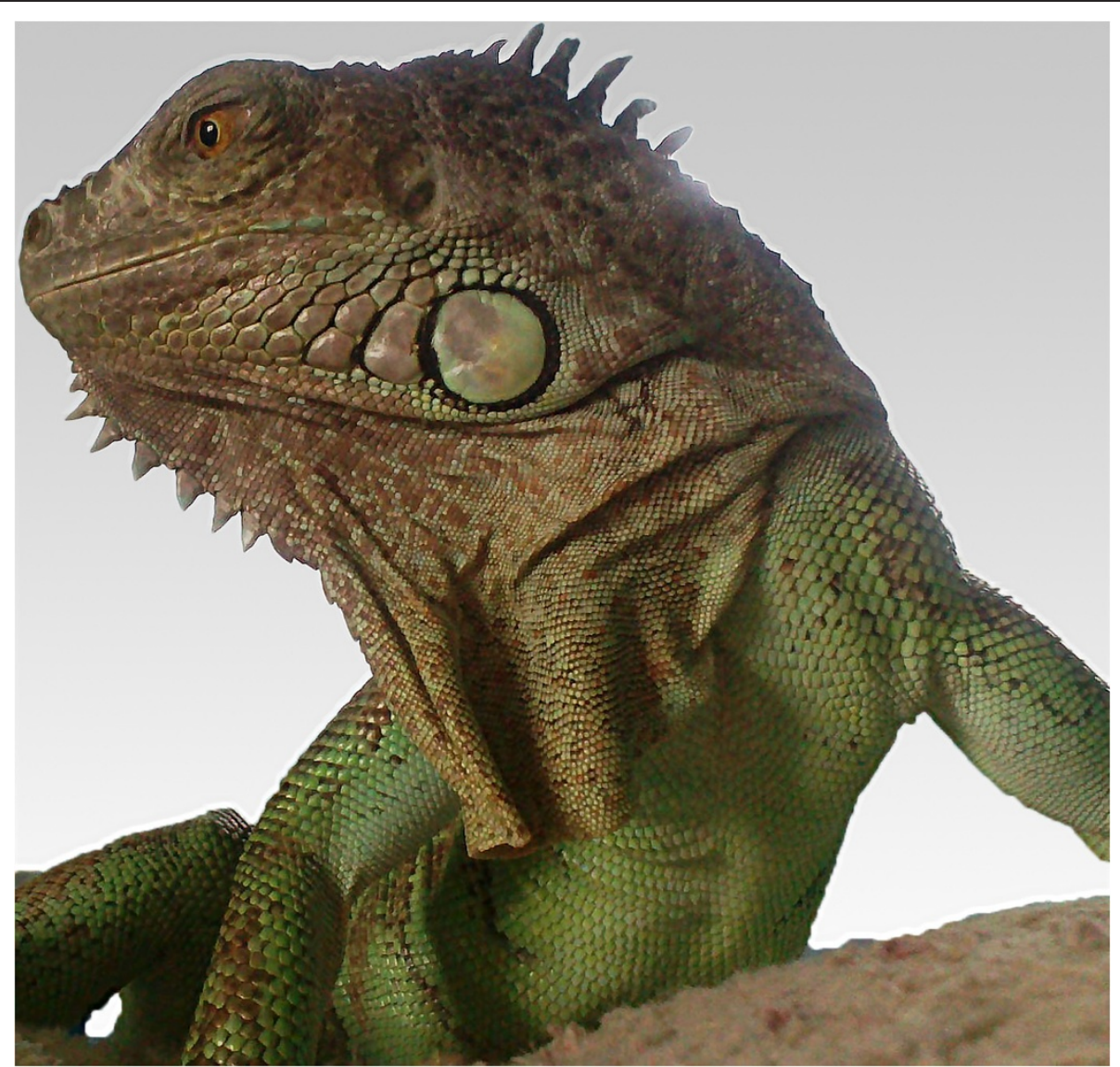

Figure 1 The infected animal: 5-year-old female green iguana (Iguana iguana). 
clinical signs, were referred to a physician for medical examination and received the same treatment. Serological investigations were not induced in the contact persons, because the physicians could not find a laboratory providing this analysis. Unfortunately, no information about follow up examinations of the contact persons is available.

\section{Isolation, identification, and molecular characterisation of} B. pseudomallei

For microbiological diagnosis, the pus from the abscess was submitted on a swab with Amies transport medium (D\&D Laborservice, Henningsdorf, Germany) to the Vet Med Labor GmbH, Ludwigsburg, Germany. The sample was cultured on MacConkey agar (BioMerieux, Nürtingen, Germany), tryptic soy agar with $5 \%$ sheep blood, chocolate agar (both BD, Heidelberg, Germany) and incubated at $36^{\circ} \mathrm{C}$ under atmosphere with $5 \% \mathrm{CO}_{2}$ for 24 hours, and afterwards for 24 hours in ambient atmosphere. Additionally, brain heart infusion enrichment broth was inoculated and sub-cultured for 24 hours on the same agar media as above. Heavy growth of metallic gleaming navel forming colonies was visible in pure culture after 48 -h incubation on all culture media. The isolate VB976100 could also be grown at $42^{\circ} \mathrm{C}$, but not at $4^{\circ} \mathrm{C}$. It showed a positive Oxidase reaction (VWR, Darmstadt, Germany) and was tested negative for catalase (Sigma-Aldrich Chemie GmbH, Munich, Germany). Short gram-negative rods with bipolar "safety pin" appearance were visible after Gram staining (Figure 2). Identification with Vitek 2 (BioMerieux) resulted in $B$. pseudomallei with $95 \%$ probability, corresponding to a very good validity of identification. The same identification result was obtained with MALDI-TOF mass spectrometry (Biotyper Microflex LT, Bruker Daltonics GmbH, Bremen,

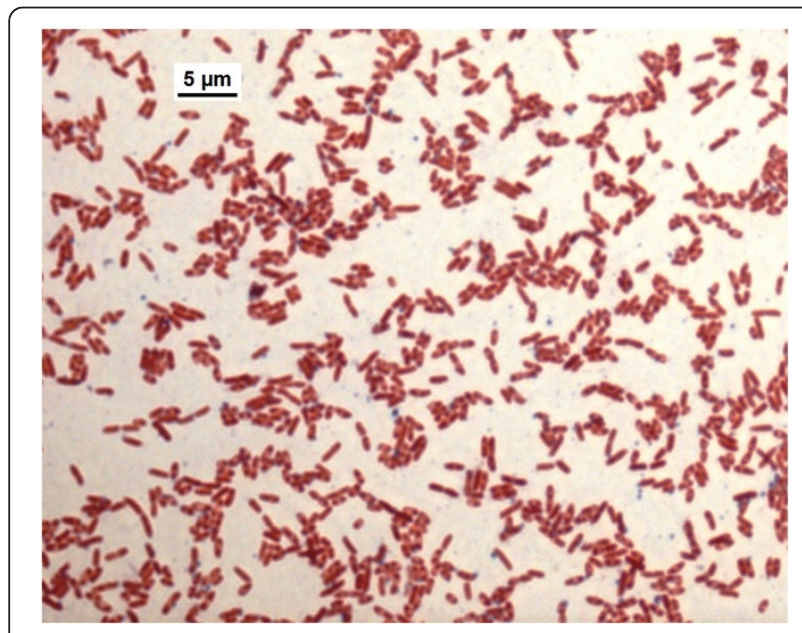

Figure 2 Gram-stain of $B$ pseudomallei isolate VB976100, phase contrast microscopy with a $100 \mathrm{x}$ oil immersion objective (Microscope Leica DM4000B).
Germany) with very high score values of $>2.600$ corresponding to highly probable species identification.

For confirmation of B. pseudomallei and further characterization, the culture was sent to the Federal Research Institute for Animal Health, Jena, Germany. The isolate VB976100 was cultured under biosafety level 3 conditions and the typical shape of colonies was seen on Ashdown-Agar [10] after $72 \mathrm{~h}$ (Figure 3).

Molecular identification was performed by real-time PCR detecting B. mallei/pseudomallei-specific sequences of the $f l i C$ gene [11] and B. pseudomallei-specific sequences of the orf 2 gene, which belongs to the type III secretion system [12].

Multi-locus sequence typing (MLST), the predominant method for molecular subtyping of B. pseudomallei was performed by comparison of 7 housekeeping genes [13]. The isolate was typed as sequence type (ST) 436 by the B. pseudomallei database of MLST.Net [14], and data have been deposited there. This ST has been only isolated in California from a patient originating from Guatemala in 2012 [15]. However, for an isolate of unknown origin, MLST alone is not adequate for determination of the geographic origin $[15,16]$. Therefore, we performed the 16S-32S internal transcribed spacer (ITS) typing [16]. For identification of the ITS type, the obtained sequence data were aligned to known ITS types A, B, C, D, E, and G, available data at GenBank (FJ981703-F981726) using the Software Geneious 6.1.8. (Biomatters Development Team). Our B. pseudomallei isolate VB976100 was typed as ITS type G (613 base pairs), and the ITS sequence was annotated and submitted to GenBank under the accession number KP069478.

\section{Conclusions}

Import of B. pseudomallei infected animals from endemic areas has been previously reported in sheep, goats and pigs from Aruba [17]. In the 1970s a B. pseudomallei outbreak was reported in the zoo of Paris, and the infection was spread to other zoos and equestrian clubs. In the course of this outbreak, termed "l'affaire du jardin des plantes", two individuals died. Imported horses from Iran or an imported panda from China were assumed as sources of infection $[18,19]$.

B. pseudomallei infections in green iguanas were already detected in California, USA, in 2007 and 2012. The bacteria were isolated from the abscess, as well as from the animal housing [8]. In both cases the isolates were typed as ST 518 and ITS type G. The authors suggested that the animals had been infected in Central America, where most imported iguanas in the USA originate from. Furthermore, these cases already highlighted the possible risk of human infections because the infection in the iguanas was not curable in spite of refractory antibiotic treatment. In one of these cases after the incubation 


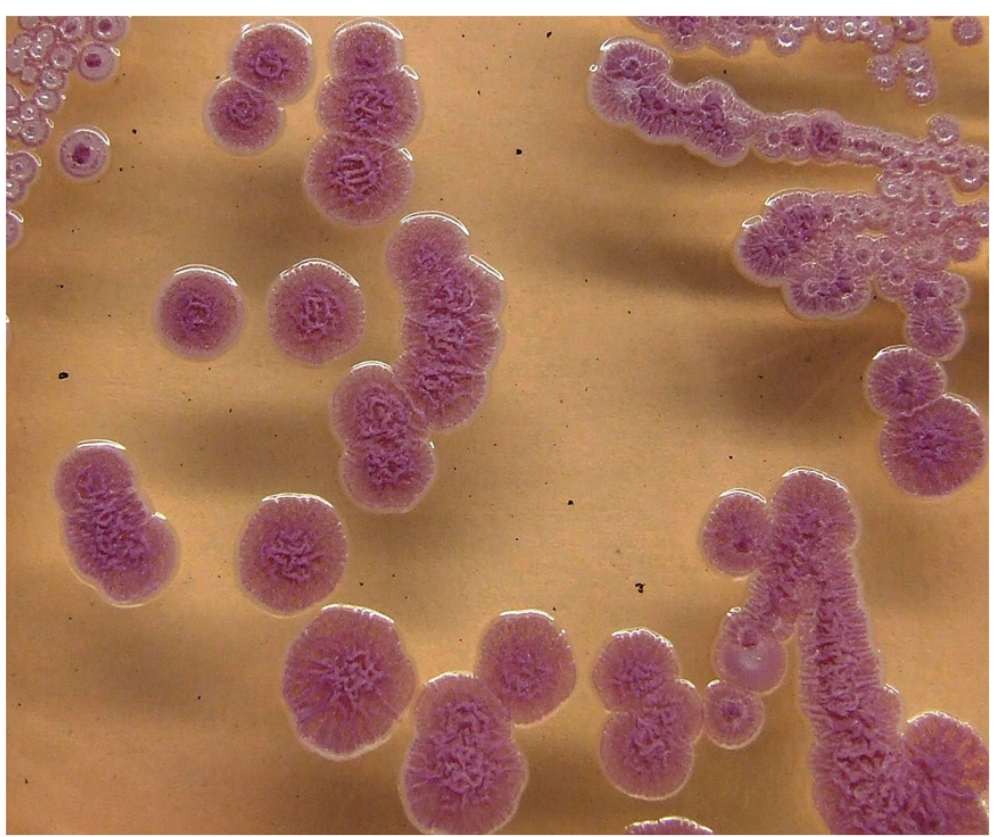

Figure 3 Colonies of B. pseudomallei isolate VB976100 on Ashdown agar showing the typical pink crinkled colonies after $72 \mathrm{~h}$ incubation.

period of around 1.5 years an abscess was diagnosed. In the clinical course of the present reported B. pseudomallei infection, the animal developed abscesses at the age of 2,4 and 5 years. However, there was no known history of any kind of trauma, which potentially could have triggered the development of abscesses [8,20]. Unfortunately, only in 2014 bacteriological examination was initiated. It is speculative but possible that after an incubation period of 2 years the first abscess was caused by a B. pseudomallei infection. These finding strongly supports the presumption, that in these animals a long incubation period is seen, bearing a risk of long not recognized phase of shedding and herewith infection risk for contact persons.

The iguana was purchased as a young animal in 2009, and unfortunately, it was not possible to determine the origin of this animal. However, the ST 436 was hitherto described for one B. pseudomallei strain, isolated in USA, originating from Guatemala [15]. On the other hand, the ITS type G of B. pseudomallei is a less common type in endemic regions, such as Australia and Southeast Asia. This type seems to be associated with isolates found only in sporadic melioidosis regions like Africa and South America [15,16]. These findings support the hypothesis that the iguana became infected in this region or in a breeding facility through contact to other infected animals originating from this area.

Our report emphasizes the risk of importation of $B$. pseudomallei into non-endemic regions through animal trade. It is possible that for patients presenting characteristic symptoms of melioidosis but with absolutely no history of travelling to endemic regions, the attending physician could misdiagnose or overlook the suspicion of melioidosis. The utility of a post exposure prophylaxis in contact persons should be discussed with the physicians. Recommendations for prophylaxis and treatment are available from Bossi et al. [21].

Personal safety can be improved by wearing eye protection and suitable gloves during sampling and treatment of possibly affected animals. For the serological investigations in non-endemic areas IgG ELISA could be useful to identify chronically infected persons [22]. Since no standardized commercial tests are available only specialized laboratories can perform such investigations.

Although we are unable to prove the source of infection in the present case, veterinarians involved in treatment of animals and competent veterinary authorities responsible for supervision of traders should be aware of this potential risk.

\section{Abbreviations}

B: Burkholderia; MLST: Multi-locus sequence typing; ITS: Internal transcribed spacer typing; ST: Sequence type; PCR: Polymerase chain reaction.

\section{Competing interests}

The authors declare that they have no competing interests.

\section{Authors' contributions}

MCE designed the study, coordinated the investigations, substantially contributed to analysis and evaluation of ITS sequence data, and wrote the manuscript. JH performed the clinical examination of the iguana, collected the samples and substantially contributed to the manuscript by drafting the clinical and epidemiological section. IS performed isolation and bacteriological identification of the B.pseudomallei isolate and substantially contributed to the manuscript by drafting the bacteriological methodological section. HEA, KM performed the MLST and ITS, and contributed substantially to evaluation and analysis of the sequence data. FM, KM, HEA have been substantially involved in 
the design of the study and discussion of results. All authors revised the manuscript critically for technical and scientific content and approved the final version.

\section{Acknowledgements}

We are grateful to K. Nitzsche, P. Marten and K. Fischer for excellent technical assistance.

\section{Author details}

'Friedrich-Loeffler-Institut, Federal Research Institute for Animal Health, Institute of Bacterial Infections and Zoonoses, Naumburger Strasse 96a, 07743 Jena, Germany. ${ }^{2}$ Animal Clinic, Bílá Hora, Cistovicka 44, 16300 Prague 6, Czech Republic. ${ }^{3}$ Vet Med Labor GmbH, Division of IDEXX Laboratories, Mörikestrasse 28/3, 71636 Ludwigsburg, Germany.

Received: 20 August 2014 Accepted: 18 November 2014 Published online: 28 November 2014

\section{References}

1. Limmathurotsakul D, Peacock SJ: Melioidosis: a clinical overview. Br Med Bull 2011, 99:125-139.

2. Katangwe T, Purcell J, Bar-Zeev N, Denis B, Montgomery J, Alaerts M, Heyderman RS, Dance DA, Kennedy N, Feasey N, Moxon CA: Human melioidosis, Malawi, 2011. Emerg Infect Dis 2013, 19(6):981-984.

3. Aardema H, Luijnenburg EM, Salm EF, Bijlmer HA, Visser CE, Van't Wout JW: Changing epidemiology of melioidosis? a case of acute pulmonary melioidosis with fatal outcome imported from Brazil. Epidemiol Infect 2005, 133(5):871-875.

4. Cuadros J, Gil H, Miguel JD, Marabe G, Gomez-Herruz TA, Lobo B, Marcos R, Anda P: Case report: melioidosis imported from West Africa to Europe. Am J Trop Med Hyg 2011, 85(2):282-284.

5. Cheng AC, Currie BJ: Melioidosis: epidemiology, pathophysiology, and management. Clin Microbiol Rev 2005, 18(2):383-416.

6. Ouadah A, Zahedi D, Perumal R: Animal melioidosis surveillance in Sabah. Internet I Vet Med 2006, 2(2). https://ispub.com/IJVM/2/2/5750

7. Sprague LD, Neubauer H: Melioidosis in animals: a review on epizootiology, diagnosis and clinical presentation. J Vet Med B Infect Dis Vet Public Health 2004, 51(7):305-320.

8. Zehnder AM, Hawkins MG, Koski MA, Lifland B, Byrne BA, Swanson AA, Rood MP, Gee JE, Elrod MG, Beesley CA, Blaney DD, Ventura J, Hoffmaster AR, Beeler ES: Burkholderia pseudomallei isolates in 2 pet iguanas, California, USA. Emerg Infect Dis 2014, 20(2):304-306.

9. Galyov EE, Brett PJ, DeShazer D: Molecular insights into Burkholderia pseudomallei and Burkholderia mallei pathogenesis. Annu Rev Microbiol 2010, 64:495-517.

10. Ashdown LR: An improved screening technique for the isolation of Pseudomonas pseudomallei from clinical specimens. Pathology 1979, 11:293-297.

11. Tomaso H, Scholz HC, Al Dahouk S, Pitt TL, Treu TM, Neubauer H: Development of $5^{\prime}$ nuclease real-time PCR assays for the rapid identification of the burkholderia mallei//burkholderia pseudomallei complex. Diagn Mol Pathol 2004, 13(4):247-253

12. Novak RT, Glass MB, Gee JE, Gal D, Mayo MJ, Currie BJ, Wilkins PP. Development and evaluation of a real-time PCR assay targeting the type III secretion system of Burkholderia pseudomallei. J Clin Microbiol 2006 44(1):85-90

13. Godoy D, Randle G, Simpson AJ, Aanensen DM, Pitt TL, Kinoshita R, Spratt BG: Multilocus sequence typing and evolutionary relationships among the causative agents of melioidosis and glanders, Burkholderia pseudomallei and Burkholderia mallei. J Clin Microbiol 2003, 41(5):2068-2079

14. Aanensen DM, Spratt BG: The multilocus sequence typing network: mlst. net. Nucleic Acids Res 2005, 33(Web Server issue):W728-W733.

15. Gee JE, Allender CJ, Tuanyok A, Elrod MG, Hoffmaster AR: Burkholderia pseudomallei type G in Western Hemisphere. Emerg Infect Dis 2014, 20(4):682-684.

16. Liguori AP, Warrington SD, Ginther JL, Pearson T, Bowers J, Glass MB, Mayo M, Wuthiekanun V. Engelthaler D, Peacock SJ, Currie BJ, Wagner DM, Keim P Tuanyok A: Diversity of 16S-23S rDNA internal transcribed spacer (ITS) reveals phylogenetic relationships in Burkholderia pseudomallei and its near-neighbors. PLoS One 2011, 6(12):e29323.
17. Sutmoller P, Kraneveld FC, Van Der Schaaf A: Melioidosis (Pseudomalleus) in sheep, goats, and pigs on Aruba (Netherland Antilles). J Am Vet Med Assoc 1957, 130(9):415-417.

18. Dance DA: Melioidosis: the tip of the iceberg? Clin Microbiol Rev 1991, 4(1):52-60

19. White NJ: Melioidosis. Lancet 2003, 361(9370):1715-1722

20. Ngauy V, Lemeshev Y, Sadkowski L, Crawford G: Cutaneous melioidosis in a man who was taken as a prisoner of war by the Japanese during World War II. J Clin Microbiol 2005, 43(2):970-972.

21. Bossi P, Tegnell A, Baka A, Van Loock F, Hendriks J, Werner A, Maidhof $H$, Gouvras $\mathrm{G}$ : Bichat guidelines for the clinical management of glanders and melioidosis and bioterrorism-related glanders and melioidosis. Euro Surveill 2004, 9(12):E17-E18.

22. Kekulé AS, Bartling C, Beyer W, Bockemühl J, Diller R, Dobler G, Essbauer S, Finke EJ, Fleischer B, Frangoulidis D, Hagen RM, Henning K, Meyer H, Neubauer H, Oehme A, Pauli G, Pfeffer M, Rakin A, Schmitz H, Splettstösser WD, Spraque LD, Tomaso H, Wölfel R, Zimmermann P: Mikrobiologischinfektiologische Qualitätsstandards (MiQ), Hochpathogene Erreger - Biologische Kampfstoffe, vol. 27. München: Elsevier; 2008:84-93.

doi:10.1186/s12917-014-0283-7

Cite this article as: Elschner et al:: Isolation of the highly pathogenic and zoonotic agent Burkholderia pseudomallei from a pet green Iguana in Prague, Czech Republic. BMC Veterinary Research 2014 10:283.

\section{Submit your next manuscript to BioMed Central and take full advantage of:}

- Convenient online submission

- Thorough peer review

- No space constraints or color figure charges

- Immediate publication on acceptance

- Inclusion in PubMed, CAS, Scopus and Google Scholar

- Research which is freely available for redistribution 